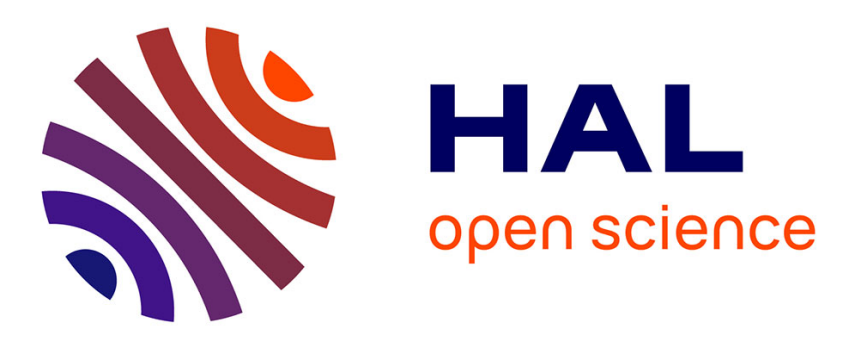

\title{
Investigation of the impact of normalization on the study of interactions between myocardial shape and deformation
}

Maxime Di Folco, Nicolas Guigui, Patrick Clarysse, Pamela Moceri, Nicolas Duchateau

\section{To cite this version:}

Maxime Di Folco, Nicolas Guigui, Patrick Clarysse, Pamela Moceri, Nicolas Duchateau. Investigation of the impact of normalization on the study of interactions between myocardial shape and deformation. FIMH 2021 - 11th International Conference on Functional Imaging and Modeling of the Heart, Jun 2021, Stanford, United States. pp.223-231, 10.1007/978-3-030-78710-3_22 . hal-03203378

\section{HAL Id: hal-03203378 https://hal.science/hal-03203378}

Submitted on 20 Apr 2021

HAL is a multi-disciplinary open access archive for the deposit and dissemination of scientific research documents, whether they are published or not. The documents may come from teaching and research institutions in France or abroad, or from public or private research centers.
L'archive ouverte pluridisciplinaire HAL, est destinée au dépôt et à la diffusion de documents scientifiques de niveau recherche, publiés ou non, émanant des établissements d'enseignement et de recherche français ou étrangers, des laboratoires publics ou privés. 


\title{
Investigation of the impact of normalization on the study of interactions between myocardial shape and deformation
}

\author{
Maxime Di Folco ${ }^{1}$, Nicolas Guigui ${ }^{2}$, Patrick Clarysse ${ }^{1}$, Pamela Moceri $^{3}$, and \\ Nicolas Duchateau ${ }^{1}$ \\ 1 Univ Lyon, Université Claude Bernard Lyon 1, INSA-Lyon, CNRS, Inserm, \\ CREATIS UMR 5220, U1294, F-69621 \\ difolco@creatis.insa-lyon.fr \\ 2 Université Côte d'Azur, Inria, Epione, Sophia-Antipolis, France \\ 3 Centre Hospitalier Universitaire de Nice, Service de Cardiologie, Nice, France - \\ Université Côte d'Azur, Faculté de médecine, Nice, France
}

\begin{abstract}
Myocardial shape and deformation are two relevant descriptors for the study of cardiac function and can undergo strong interactions depending on diseases. Manifold learning provides low dimensional representations of these high-dimensional descriptors, but the choice of normalization can strongly affect the analysis. Besides, whether the shape normalization should include a scale factor is still an open question. In this paper, we investigate the influence of normalization choices on the study of the interactions between cardiac shape and deformation using Multiple Manifold Learning, a dimensionality reduction method that considers inter- and intra-descriptors link between samples. By studying the main variations of two different shape normalizations (one including scaling, the other one not) we observed that the scaled normalization concentrates variations of a given physiological characteristic on only one mode. The influence of the associated choice of the deformation normalization was evaluated by quantifying differences between the estimated low-dimensional spaces (one for each choice against a combination of both), revealing potential analysis biases that may arise depending on such choices.
\end{abstract}

Keywords: Cardiac imaging - manifold learning $\cdot$ myocardial strain . heart shape.

\section{Introduction}

Medical imaging can provide high-dimensional descriptors of an organ's shape and function that are very complementary to the measurements used in clinical routine. However, identifying the appropriate descriptor for a particular diagnostic situation and finding an understandable representation of it is not an easy task due to the complexity of the anomalies that can co-exist.

Myocardial shape, considered at End-Diastole (ED), and deformation are two relevant descriptors to assess the cardiac function, but these are often reduced 
to scalars in clinical routine (such as volumes or ejection fraction, respectively). However, these descriptors can undergo strong structural [1] or disease-related interactions 2 . 3 .

Representation learning techniques can offer statistically-relevant and simplified views of a population or disease, but do not explicitly account for the interaction between descriptors. Within the field of manifold learning, Multiple Manifold Learning (MML) [4], generalized in 5] not only attempts to find a suitable latent space associated to a given descriptor, but also conditions the link between the representations associated to different descriptors.

Nonetheless, these methods still depend on the choice of descriptors and how these are normalized across a population. When analyzing shape features in a population, whether shape normalization should include scaling or volume invariance is still an open question. Besides, in computational anatomy, the transport of data from an individual's mesh to a reference template should be handled carefully $[6]$, and the effect of scaling or volume differences on the transported data are still debated [7, 8].

In this paper, we explore the influence of different normalization choices on the representation of myocardial shape and deformation while considering their interactions with non-linear manifold learning. We specifically examined a population of meshes representing the Right Ventricle (RV) from controls and patients with volume-overload, two conditions that affect differently shape and deformation depending on the pathology. We compared two different shape features and three strain normalization strategies, which correspond respectively to a rigid shape matching and no rescaling of the strain values, or finer shape changes with potential effect on the strain patterns.

\section{Methods}

\subsection{Data and descriptors}

Data: We processed a database of 110 meshes of RV extracted from 3D echocardiography using the commercial software 4D-RV Function 2.0 (TomTec Imaging Systems GmbH, Germany). Point-to-point correspondences are ensured between the meshes, each containing 1587 cells and 822 points (valves excluded). The database consists of patients suffering congenital diseases with RV volume overload (Atrial Septal Defect (ASD) and Tetralogy of Fallot (ToF)), and 55 age- and sex-matched controls. In the following, we represent the myocardial shape and deformation using high-dimensional descriptors at each point of the RV mesh.

Shape descriptors: In order to align the meshes spatially, a Procrustes analysis is applied with a rigid transformation (with no scale factor) and a similarity transformation (with a scale factor). This difference in normalization on the population will affect the shape and our analysis. Indeed, we characterize shape differences through a 3D vector between each point of a given individual mesh and its corresponding point on the reference. This reference was obtained by 


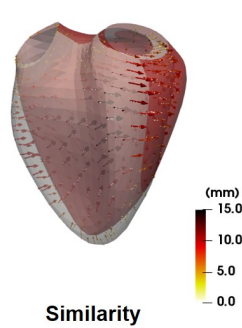

(a) Shape features
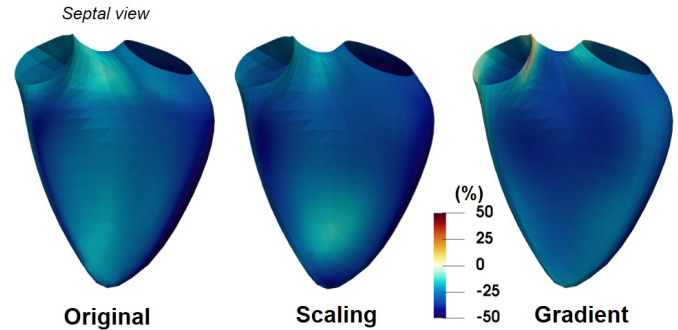

(b) Deformation features

Fig. 1: (a) Distance vector field ( $\mathrm{mm}$ ) colored by magnitude between a sample and the reference mesh (in red) for similarity and rigid transformations. (b) Average area strain (\%) for the original, scaling and gradient deformation features.

a Procrustes alignment only of the controls to encode shape normality. In the following, we denote this shape descriptor (of dimensions $822 \times 3$ ) either rigid or similarity depending in the type of Procrustes alignment used (Fig 1a).

Deformation descriptors: The deformation descriptor is the area strain corresponding to the relative area change of each mesh cell (in \%) between ED and End-Systole (ES). It quantifies the local deformation of the RV surface with a high-dimensional descriptor of dimensions $822 \times 1$. We first computed this descriptor on the original population, it is denoted original.

We also applied a normalization procedure in order to disentangle this descriptor from the organ's shape. The normalization procedure relies on the parallel transport of deformations in the diffeomorphic registration setting, with a geometric representation of deformations as elements of the group of diffeomorphisms endowed with a right-invariant Riemannian metric [6 9]. In order to define a volumeinvariant normalization, we used the metric proposed in [7]. It involves normalizing each shape so that its volume matches that of the reference shape. Two projection strategies were used, one by scaling around the barycentre of each shape, and the other one by following the flow of the gradient of the volume function. They are denoted respectively scaling and gradient (Fig.1b). The former may be biased if the center of mass of the shapes is unnatural, while the latter depends on the choice of the embedding metric, in our case the Large Deformation Diffeometric Metric Mapping (LDDMM) metric, to define the gradient.

\subsection{Manifold learning}

Given $K$ subjects and one high-dimensional descriptor for shape $\mathbf{X}^{\mathbf{S}}=\left[\mathbf{x}_{1}^{S}, \cdots, \mathbf{x}_{K}^{S}\right]^{T}$ $\in \mathbb{R}^{K \times M_{S}}$ and another one for deformation $\mathbf{X}^{\mathbf{D}}=\left[\mathbf{x}_{1}^{D}, \cdots, \mathbf{x}_{K}^{D}\right]^{T} \in \mathbb{R}^{K \times M_{D}}$ $\left(M_{D}\right.$ and $M_{S}$ are the number of input dimensions, here 822 and $2466=822 \times 3$ ), 
we jointly represented each descriptor and the interactions between their latent space using the MML algorithm. We first estimated the following affinity matrix:

$$
\mathbb{W}=\left[\begin{array}{cc}
\mathbf{W}^{S} & \mu \mathbf{M} \\
\mu \mathbf{M}^{T} & \mathbf{W}^{D}
\end{array}\right]
$$

$\mathbb{W}$ is composed of the sub-matrices $\mathbf{W}^{S}$ and $\mathbf{W}^{D}$ obtained by spectral embedding on the shape and deformation descriptors independently, which represent the intra-descriptor correspondences, and the matrix $\mathbf{M}$ (weighted by a scalar value $\mu>0$ ) which encodes the inter-descriptor correspondences:

$$
\begin{aligned}
W_{i j}^{S} & =\exp \left(-\left\|\mathbf{x}_{\mathbf{i}}{ }^{S}-\mathbf{x}_{\mathbf{j}}{ }^{S}\right\|^{2} / \sigma^{2}\right) \\
W_{i j}^{D} & =\exp \left(-\left\|\mathbf{x}_{\mathbf{i}}{ }^{D}-\mathbf{x}_{\mathbf{j}}{ }^{D}\right\|^{2} / \sigma^{2}\right)
\end{aligned} \quad M_{i j}=\frac{<\mathbf{w}_{i}^{S}, \mathbf{w}_{j}^{D}>}{\left\|\mathbf{w}_{i}^{S}\right\|\left\|\mathbf{w}_{j}^{D}\right\|}
$$

where $\sigma$ is the width of the kernel used for spectral embedding, determined as the average distance between a sample and its $k_{\sigma}$ nearest neighbors, and $\mathbf{w}_{i}^{S}$ $\left(\right.$ resp. $\mathbf{w}_{i}^{D}$ ) is the $i$-th row of the affinity matrix $\mathbf{W}^{S}\left(\right.$ resp. $\left.\mathbf{W}^{D}\right)$. The matrix $\mathbf{M}$ is sparsified afterwards by setting to zero each element that does not belong to the $k_{M}$ closest neighbors of each sample (determined as the $k_{M}$ highest values in each row of the affinity matrix).

MML aims at minimizing:

$$
\Phi(\mathbf{Y})=\sum_{i, j=1}^{K}\left\|\mathbf{y}_{i}^{S}-\mathbf{y}_{j}^{S}\right\|^{2} W_{i j}^{S}+\sum_{i, j=1}^{K}\left\|\mathbf{y}_{i}^{D}-\mathbf{y}_{j}^{D}\right\|^{2} W_{i j}^{D}+\mu \sum_{i, j=1}^{K}\left\|\mathbf{y}_{i}^{S}-\mathbf{y}_{j}^{D}\right\|^{2} M_{i j},
$$

where $\mathbf{Y}^{\mathbf{S}}=\left[\mathbf{y}_{1}^{S}, \cdots, \mathbf{y}_{K}^{S}\right]^{T} \in \mathbb{R}^{K \times N}$ and $\mathbf{Y}^{\mathbf{D}}=\left[\mathbf{y}_{1}^{D}, \cdots, \mathbf{y}_{K}^{D}\right]^{T} \in \mathbb{R}^{K \times N}$ respectively stand for the latent spaces coordinates of the shape and deformation descriptors, $N$ being the number of latent dimensions retained. They are obtained by computing the normalized graph Laplacian and then using Diffusion Maps as described in [10|. The first half of the eigenvectors of $\mathbb{W}$ encodes the data from the first descriptor (shape, $K$ first eigenvectors), while the second half corresponds to the second descriptor (deformation, $K$ last eigenvectors). Thus, MML provides one latent space for each descriptor, meaning that we have two low-dimensional latent spaces, one representing the shape and the other one the deformation.

\section{$3 \quad$ Experiments and results}

The following section describes the application of MML to the entire population (except one control due to obvious mesh defects), with the hyperparameters: $k_{\sigma}=10, k_{M}=10$ and $\mu=1$. These parameters have been determined empirically from the energy defined in Eq.2

Figure 2 shows the obtained deformation latent spaces for the different combinations of shape and deformation descriptors. Note that for the rigid-gradient latent space, the second and third dimensions are displayed. Indeed, with spectral embedding several eigenvectors may encode the same spatial direction and make 

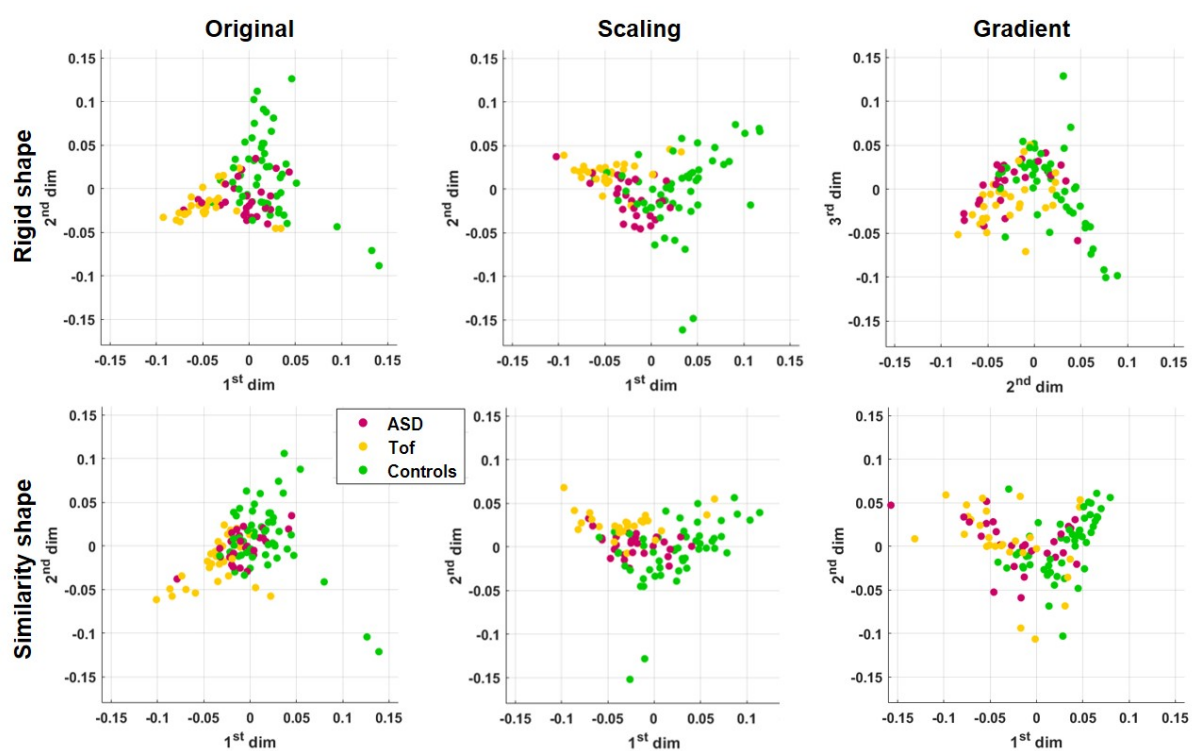

Fig. 2: Latent spaces encoding deformation, provided by MML for the 6 different normalization strategies.

some dimensions redundant [11]. This is the case with the first and the second dimensions here.

ASD samples are more centered and close from each other using the original and scaling deformation descriptors compared to the gradient descriptors. The different populations seem more separated for these strategies especially the ToF and controls. However, the distribution of samples in the latent spaces is not enough to assess the difference between normalization strategies. In the next section, we explore the influence of the shape normalization on the main variations encoded in the latent spaces.

\subsection{Shape descriptors}

For the similarity-original and rigid-original latent spaces encoding shape, we reconstructed the main characteristics encoded from $-2 \sigma$ to $2 \sigma$ along the first dimensions ( $\sigma$ being the standard deviation along the considered dimension), using multi-scale regression as described in [12]. Visualization consisted of the shapes obtained after adding the reconstructed vector fields to the reference shape. We then compared the different normalization strategies by defining some shape characteristics on the reconstructed meshes: the intra-valve distance, the width, the depth and the length of the meshes as depicted in Fig 3a and also the volume. Each distance was computed between chosen points on the mesh for each measure, which are straightforward to obtain for different subjects as point-to-point correspondences are known. 


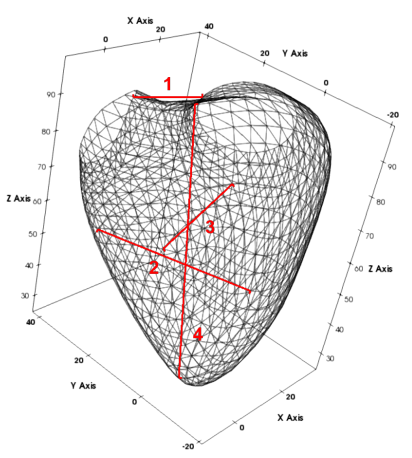

(a)

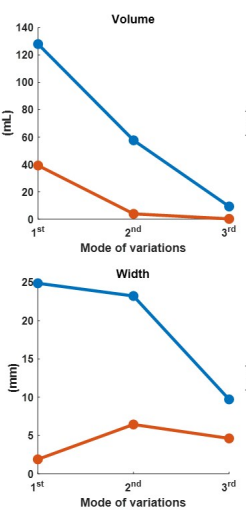

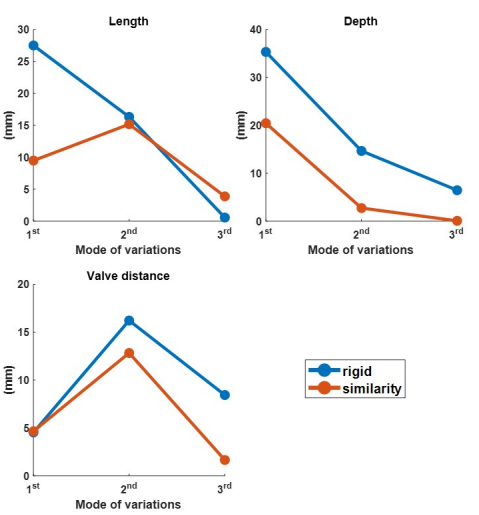

(b)

Fig. 3: Variations of 5 different shape characteristics defined on each mesh, when moving from $-2 \sigma$ to $+2 \sigma$ along a given dimension of the latent space with rigid and similarity normalizations. The characteristics are defined in (a) with (1) valve distance, (2) RV width, (3) depth and (4) length.

Figure $3 \mathrm{~b}$ shows the relative change of a given measure when moving between $-2 \sigma$ and $+2 \sigma$ for the first three modes of variations. We can observe that the similarity strategy concentrates volume and depth variations on the first mode, while finer changes predominate on the next modes, such as length and valve distance on the second mode. For the rigid descriptor, substantial variations of volume, length and width happened on the first two modes. The depth and the valve distance variations are mainly on one mode (first and second respectively).

\subsection{Deformation descriptors}

In this section, we evaluated the influence of the normalization choice for the deformation descriptors. We first applied MML on two combinations of the deformation descriptors (original/scaling or original/gradient) and each shape normalization. These spaces are considered as reference and we evaluated the difference to the corresponding latent space displayed in Fig. 2. By comparing them to a combination of both descriptors equally weighted (to do so, we replaced the submatrix $\mathbf{W}^{D}$ by the arithmetic average of the associated affinity matrices), a predominant normalization may appear. Differences between the latent spaces were quantified by the point-to-point differences between samples. As these may be noisy, a 2D kernel regression was used on the first two dimensions of the latent spaces to smooth out the differences and better visualize trends for the whole population or specific subgroups. The regression involved a $18 \times 18$ grid equally distributed between -0.1 and 0.1 for the first two dimensions. To leverage the contribution of samples in less populated regions, we encoded the density around each sample as the opacity of arrows. 

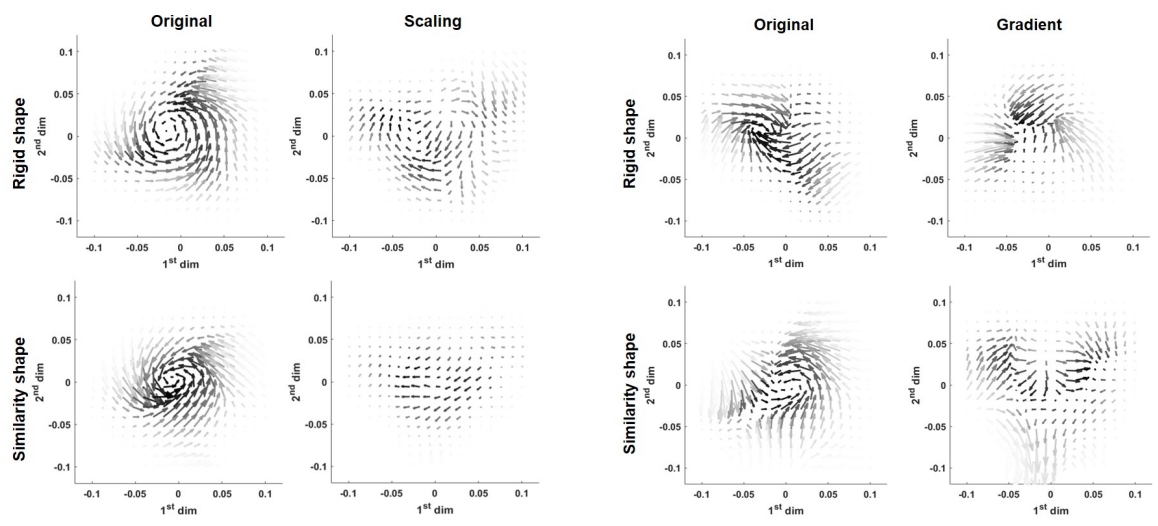

(a)

(b)

Fig. 4: Vector field encoding the local distances in the latent spaces between a combination of (a) original/scaling and (b) original/gradient. The opacity reflects to the estimated density at each point of the grid.

Figure 4a shows the results for the original/scaling combination. The original deformation vector field seems to rotate around the center for the latent space, for the two shape normalizations. Few changes are observed for the scaling deformation, even though the rigid + scaling exhibits a reduced rotation. Concerning the combination of original/gradient displayed in Fig $4 \mathrm{~b}$ rigid + original and rigid + gradient show substantial changes that seem to be in opposite directions. similarity + original also exhibits a rotation and changes occur for similarity + gradient mainly in a low density zone.

\section{Discussion and conclusion}

In this paper, we investigated the influence of the normalization on the study of the interactions between the myocardial shape and the cardiac deformation using MML.

We evaluated the differences between two shape normalization strategies (rigid and similarity with a scaling factor), through the main modes of variations of the population. Several measurements on the meshes show that the variations are mainly concentrated on one mode for similarity (first mode for volume and depth, second mode for length and valve distance), unlike rigid where substantial variations jointly appear on several modes, except for the valve distance. Specific latent dimensions provide more understandable variations, which is an asset for the study of the population intra-variability.

Then, we assessed the influence of the normalization on the deformation feature. The two normalizations (scaling and gradient) predominate on original (a rotation appeared on every combination with no normalization). This rotation might correspond to the bias introduced by an improper pair of shape and 
deformation normalizations. Indeed, it can be expected that applying a volumerelated normalization on only one of the features can introduce such a bias. The similarity descriptor has more affinity to the normalized deformation descriptor (less change in deformation field) compared to the rigid.

As we focus our work on the study of interactions between cardiac shape and deformation, we would like to prevent potential biases introduced by the normalization. These experiments provided insights into the influence of the shape normalization by itself, and the choices of pairs of shape and deformation normalizations. They revealed differences between the two strategies of normalization for the deformation feature. However the choice of an optimal strategy is likely to depend on the application and the associated data. Other state-ofthe-art strategies to normalize the descriptors will be explored in future work.

Acknowledgements: The authors acknowledge the support from the French ANR (LABEX PRIMES of Université de Lyon [ANR-11-LABX-0063] within the program "Investissements d'Avenir" [ANR-11-IDEX-0007], and the JCJC project "MIC-MAC" [ANR-19-CE45-0005]), and the EEA doctoral school. This work was also partially funded by the ERC grant Nr. 786854 G-Statistics from the European Research Council under the European Union's Horizon 2020 research and innovation program, and by the French government through the 3IA Côte d'Azur Investments ANR-19-P3IA-0002 managed by the National Research Agency. The authors are grateful to the OPAL infrastructure from Université Côte d'Azur for providing resources and support.

\section{References}

1. B Bijnens, M Cikes, C Butakoff, et al. Myocardial motion and deformation: What does it tell us and how does it relate to function? Fetal Diagn Ther, 32:5-16, 2012.

2. P Moceri, N Duchateau, S Gillon, et al. Three-dimensional right ventricular shape and strain in congenital heart disease patients with right ventricular chronic volume loading. Eur Heart J Cardiovasc Imaging, 2020. In press.

3. J Sanz, D Sánchez-Quintana, E Bossone, et al. Anatomy, function, and dysfunction of the right ventricle. J Am Coll Cardiol, 73:1463-82, 2019.

4. J Ham, DD Lee, and LK Saul. Semisupervised alignment of manifolds. Proc. AISTATS, 120:120-7, 2005.

5. JR Clough, D Balfour, G Cruz, et al. Weighted manifold alignment using wave kernel signatures for aligning medical image datasets. IEEE Trans Pattern Anal Mach Intell, 42:988-97, 2019.

6. M Lorenzi and X Pennec. Efficient Parallel Transport of Deformations in Time Series of Images: From Schild to Pole Ladder. J Math Imaging Vis, 50:5-17, 2014.

7. M Niethammer and F Vialard. Riemannian metrics for statistics on shapes: Parallel transport and scale invariance. Proc. MFCA-MICCAI, 2013.

8. N Guigui, P Moceri, M Sermesant, et al. Cardiac motion modeling with parallel transport and shape splines. Proc. ISBI, 2021. In press.

9. L Younes. Shapes and Diffeomorphisms, volume 171 of Applied Mathematical Sciences. Springer, Berlin, Heidelberg, 2019.

10. RR Coifman and S Lafon. Diffusion maps. Appl Comput. Harmon Anal, 21:5-30, 2006. 
This a pre-print version.

The final document will be available at http://www.springerlink.com

11. B Nadler, S Lafon, R Coifman, et al. Diffusion Maps - a probabilistic interpretation for spectral embedding and clustering algorithms. In Principal Manifolds for Data Visualization and Dimension Reduction, pages 238-260. Springer, Berlin, Germany, 2008.

12. N Duchateau, M De Craene, M Sitges, et al. Adaptation of multiscale function extension to inexact matching: Application to the mapping of individuals to a learnt manifold. Proc. SEE-GSI, LNCS, 8085:578-86, 2013. 